\title{
ALKBH5 Inhibits Pancreatic Cancer Motility by Decreasing Long Non-Coding RNA KCNK15-AS1 Methylation
}

\author{
Yuan $\mathrm{He}^{\mathrm{a}, \mathrm{b}, \mathrm{c}}$ Hao Hu $\mathrm{u}^{\mathrm{a}, \mathrm{d}}$ Yandong Wang ${ }^{\mathrm{a}, \mathrm{e}}$ Hao Yuan ${ }^{\mathrm{a}}$ Zipeng Lu ${ }^{\mathrm{a}}$ \\ Pengfei $\mathrm{Wu}^{\mathrm{a}}$ Dongfang Liu ${ }^{\mathrm{a}}$ Lei Tian ${ }^{\mathrm{a}}$ Jie Yin ${ }^{\mathrm{a}}$ Kuirong Jiang ${ }^{\mathrm{a}}$ \\ Yi Miao ${ }^{\mathrm{a}}$
}

\begin{abstract}
${ }^{a}$ Department of General Surgery, Pancreas Centre, the First Affiliated Hospital of Nanjing Medical University, Nanjing, bDepartment of General Surgery, Heping Hospital, Changzhi Medical College, Changzhi, 'Department of General Surgery, Huai'an People's Hospital Affiliated to Xuzhou Medical University, Huai'an, dDepartment of Hepatopancreatobiliary Center, The Third Hospital Affiliated to Nantong University, ${ }^{\text {DDepartment }}$ of General Surgery, The Second People's Hospital of Wuhu, Wuhu, P. R. China
\end{abstract}

\section{Key Words}

Long non-coding RNA • Pancreatic cancer $\cdot$ KCNK15-AS1 $•$ ALKBH5 $\bullet \mathrm{M}^{6} \mathrm{~A}$

\begin{abstract}
Background/Aims: Mounting evidence suggests that epitranscriptional modifications regulate multiple cellular processes. $N^{6}$-Methyladenosine $\left(\mathrm{m}^{6} \mathrm{~A}\right)$, the most abundant reversible methylation of mRNA, has critical roles in cancer pathogenesis. However, the mechanisms and functions of long non-coding RNA (IncRNA) methylation remain unclear. Pancreatic cancer resulted in 411,600 deaths globally in 2015. By the time of pancreatic cancer diagnosis, metastasis has often occurred in other parts of the body. The present study sought to investigate IncRNA m6A modification and its roles in pancreatic cancer. Methods: Differential expression between cancer cells and matched normal cells was evaluated to identify candidate IncRNAs. The IncRNA KCNK15-AS1 was detected in cancer tissues and various pancreatic cells using RT-qPCR. KCNK15-AS1 was transfected into cells to explore its role in migration and invasion. Then, $\mathrm{m}^{6} \mathrm{~A}$ RNA immunoprecipitation was performed to detect methylated KCNK15AS1 in tissues and cells. Epithelial-mesenchymal transition (EMT) markers were used to evaluate KCNK15-AS1-mediated EMT processes. Results: KCNK15-AS1 was downregulated in pancreatic cancer tissues compared with paired adjacent normal tissues. KCNK15-AS1 inhibited migration and invasion in MIA PaCa-2 and BxPC-3 cells. Furthermore, total RNA methylation in cancer cells was significantly enriched relative to that in immortalized human pancreatic duct epithelial (HPDE6-C7) cells. In addition, the $\mathrm{m}^{6} \mathrm{~A}$ eraser ALKBH5 was downregulated in cancer cells, which can demethylate KCNK15-AS1 and regulate KCNK15-AS1-mediated cell motility. Conclusion: Our results have revealed a novel mechanism by which ALKBH5 inhibits pancreatic cancer motility by demethylating IncRNA KCNK15-AS1, identifying a potential therapeutic target for pancreatic cancer.

Y. He, H. Hu, Y. Wang and H. Yuan contributed equally to this work.

Dr. Kuirong Jiang

and Dr. Yi Miao

Dept. of General Surgery, Pancreas Centre, the First Affiliated Hospital with Nanjing

Med. Univ. 300 Guangzhou Road, Nanjing, (PR China)

Tel.+86-025-68136891, E-Mail jiangkuirong@njmu.edu.cn; miaoyi@njmu.edu.cn
\end{abstract}




\section{Cellular Physiology Cell Physiol Biochem 2018;48:838-846 \begin{tabular}{l|l} 
DOI: 10.1159/000491915 & Ond Biochemistry \\
Published online: July 20, 2018 & $\begin{array}{l}\text { 2018 The Author(s). Published by S. Karger AG, Basel } \\
\text { www.karger.com/cpb }\end{array}$
\end{tabular} \\ ALKBH5 Mediates Pancreatic Cancer via LncRNA}

\section{Introduction}

Pancreatic cancer is one of the most aggressive types of cancer and is the sixth leading cause of cancer-related deaths in China [1] and the seventh worldwide [2], respectively. It causes 330, 000 deaths annually and constitutes a notable global health problem [2]. Pancreatic cancer has an extremely poor prognosis, with a median survival of 5 to 8 months (less than $10 \%$ survival rate). Pancreatic cancer develops with variable symptoms, local invasiveness, or metastases to distant sites in its early stages [3]. Accordingly, it is important to investigate the mechanisms that contribute to metastasis in pancreatic cancer.

Long non-coding RNAs (lncRNAs) are non-coding RNAs comprising more than 200 nucleotides that lack protein coding capacity. Mounting evidence has demonstrated that lncRNAs play important roles in cancer cell proliferation, apoptosis, and metastasis. Although HOTAIR [4], HOTTIP [5], and MALAT1 [6] have been reported in pancreatic cancer studies, the mechanisms by which lncRNAs regulate pancreatic cancer require further exploration.

More than 100 RNA modifications have been reported [7], but there are few reports on lncRNA modifications. $N^{6}$-methyladenosine $\left(\mathrm{m}^{6} \mathrm{~A}\right)$ modification is the most common internal modification in eukaryotic mRNAs [8]. Two recently identified mammalian RNA demethylases, the fat mass and obesity-associated protein (FTO) and alkylation repair homolog protein 5 (ALKBH5), have demonstrated that the $\mathrm{m}^{6} \mathrm{~A}$ methylation modification is a dynamic and reversible process. However, the mechanism by which FTO and ALKBH5 regulate IncRNA methylation requires further investigation.

In this study, we demonstrate that the lncRNA KCNK15-AS1 is significantly downregulated in pancreatic cancer, revealing its inhibitory functions on cell migration and invasion. Additionally, the $\mathrm{m}^{6} \mathrm{~A}$ eraser ALKBH5 was found to be downregulated in cancer tissues, where it could demethylate KCNK15-AS1 and regulate KCNK15-AS1 expression. ALKBH5 was also found to be involved in KCNK15-AS1-mediated cell migration and invasion. In the future, ALKBH5-KCNK15-AS1 might serve as a therapeutic target for patients with pancreatic cancer.

\section{Materials and Methods}

Clinical samples

Fresh frozen pancreatic cancer tissues and their adjacent normal tissues were obtained from the First Affiliated Hospital of Nanjing Medical University. The samples were obtained within 10 min after tumor excision and immediately stored at $-80^{\circ} \mathrm{C}$ until use in experiments. Written informed consent was obtained from all patients, and this study was approved by the Ethics Committee of the hospital (2017-SR-171).

\section{Microarray analysis}

Total RNA was extracted from three pancreatic cancer tissues and paired adjacent normal tissues, and the expression profile of 30, 584 human IncRNAs was determined using Arraystar Human LncRNA Microarray V3.0. Volcano plot filtering, set at a threshold of $\geq 2.0$-fold, was used to screen for lncRNAs that exhibited significantly different $(P<0.05$; unpaired $t$-test) expression levels in the two study groups.

\section{Cell culture and cell transfection}

Human pancreatic cancer cell lines (MIA-PaCa-2 and BxPC-3) and human embryonic kidney cells (293T) were purchased from the American Type Culture Collection (ATCC, Manassas, VA), and the immortal human pancreatic duct epithelial cell line (HPDE6-C7), which is considered a normal pancreatic cell line, was obtained from the Pancreas Institute, Nanjing Medical University. HPDE6-C7, MIA-PaCa-2, and BxPC-3 cells were maintained in Dulbecco's modified Eagle's medium supplemented with $10 \%$ fetal bovine serum (FBS), and 1\% penicillin/streptomycin. All cell lines were cultured in an atmosphere of $5 \% \mathrm{CO}_{2}$ and $99 \%$ relative humidity at $37^{\circ} \mathrm{C}$. Lipofectamine $2000^{\mathrm{TM}}$ was applied to conduct transfections in accordance with the manufacturer's guidelines (Invitrogen, Carlsbad, CA). 


\section{Cellular Physiology Cell Physiol Biochem 2018;48:838-846 \\ \begin{tabular}{l|l} 
and Biochemistry Published & $\begin{array}{l}\text { DOI: 10.1159/00491915 } 2018 \text { The Author(s). Published by S. Karger AG, Basel } \\
\text { www.karger.com/cpb }\end{array}$ \\
\hline
\end{tabular}}

ALKBH5 Mediates Pancreatic Cancer via LncRNA

\section{RNA isolation and reverse transcription}

Total RNA was isolated from cells and tissues using TRIzol reagent (Invitrogen) according to the manufacturer's instructions. The NanoDrop ND2000 (Thermo Scientific Inc., Waltham, MA) was used to determine RNA purity and to quantify RNA concentration. A First Strand cDNA Synthesis Kit (TaKaRa, Beijing, People's Republic of China) was used to perform reverse transcription.

Real-time quantitative polymerase chain reaction

Real-time quantitative polymerase chain reaction (RT-qPCR) primers were obtained from GenScript (Nanjing, People's Republic of China), and the sequences are listed in Table 1. According to the manufacturer's instructions, RT-qPCR was performed using the SYBR Prime-Script RT-PCR Kit (Takara Biomedical Technology (Beijing) Co., Ltd., Beijing, China) with a Roche LightCycler 96 fluorescent quantitative PCR system (Roche, Mannheim, Germany). The reaction started at $95^{\circ} \mathrm{C}$ for $5 \mathrm{~min}$, followed by 40 cycles of $95^{\circ} \mathrm{C}$ for $30 \mathrm{~s}, 59^{\circ} \mathrm{C}$ for $30 \mathrm{~s}$, and $72^{\circ} \mathrm{C}$ for $30 \mathrm{~s}$. Actin was used as the internal control to normalize qPCR results. Relative gene expression levels were measured using cycle threshold (CT) values in the $\Delta \Delta \mathrm{CT}$ calculation $[4]$.

\section{Cell migration and invasion assay}

Transwell assays were conducted with an $8-\mu \mathrm{m}$ pore size insert with or without Matrigel Invasion Chambers (Millipore Corporation, Burlington, MA) to evaluate the properties of migration or invasion of pancreatic cells. Then, $48 \mathrm{~h}$ post-transfection, single-cell suspensions were seeded into the upper chamber, and the lower chamber was filled with $500 \mu \mathrm{L}$ of Dulbecco's Modified Eagle's Medium containing 10\% FBS. After the indicated incubation time, the non-migrating or invading cells were wiped from the upper surface of the membranes, and cells were stained with $2 \%$ crystal violet solution after fixation in methanol. Images of migrated cells in each well were captured and counted in random fields using a microscope. Each experiment was conducted in triplicate.

\section{Western blotting and antibodies}

Total protein $(60 \mu \mathrm{g})$ was subjected to SDS-PAGE and transferred to a $0.45-\mu \mathrm{m}$ PVDF membrane (Millipore Corporation). Antibodies against ALKBH5 (Abcam, Cambridge, MA; 1:100) and actin (Santa Cruz Biotechnology, Santa Cruz, CA; 1:2000) were used. Membranes were incubated overnight at $4^{\circ} \mathrm{C}$ with primary antibodies diluted 1:1, 000 to 1:10, 000 according to the manufactures' instructions, and proteins were detected with a peroxide LumiGLO reagent (Cell Signaling Technology, Danvers, MA).

\section{RNA immunoprecipitation}

RNA immunoprecipitation (RIP) experiments were performed using the Magna RIP RNA-Binding Protein Immunoprecipitation Kit (Millipore Corporation). Antibodies against $\mathrm{m}^{6} \mathrm{~A}$ (Abcam) or control proteins were diluted to 1:50. Total RNA (input control) and precipitation with the isotype control (IgG) for each antibody were assayed simultaneously. The co-precipitated RNAs were detected by qRT-PCR.

\section{Confocal microscopy}

Cells were fixed with $4 \%$ paraformaldehyde in PBS at room temperature for $10 \mathrm{~min}$ and permeabilized with $0.1 \%$ Triton-X and $1 \%$ BSA in PBS for $30 \mathrm{~min}$ at room temperature. DNA was stained using 4,6-diamidino-2phenylindole (DAPI). KCNK15-AS1 antisense probe hybridization was performed at $37^{\circ} \mathrm{C}$ for about $16 \mathrm{~h}$ to detect KCNK15-AS1. The sequences of probes are listed in Table 2. Imaging was performed using a Zeiss Axiovert 200M laser scanning confocal microscope (Carl Zeiss AG, Oberkochen, Germany).
Table 1. Primers used for RT-qPCR

\begin{tabular}{lc}
\hline Primer & \multicolumn{2}{c}{ Sequence } \\
\hline ALKBH5 & F: 5'-TGAGCACAGTCACGCTTCCC-3' \\
& R: 5'-TCCGTGTCCTTCTTTAGCGACTC-3' \\
& F: 5'- TAAAGCCATCACCAGGACAAC-3' \\
KCNK15-AS1 & R: 5'- ATACATTTGGCTCCCATGGCTA-3' \\
& F: 5'- GTGGCCGAGGACTTTGATTG-3'
\end{tabular}

R: 5'- CCTGTAACAACGCATCTCATATT-3' 


\section{Cellular Physiology Cell Physiol Biochem 2018;48:838-846 and Biochemistry Publis.1159/000491915 $2018 \quad \begin{aligned} & \text { (c) } 2018 \text { The Author(s). Published by S. Karger AG, Basel } \\ & \text { www.karger.com/cpb }\end{aligned}$ ALKBH5 Mediates Pancreatic Cancer via LncRNA}

Statistical analyses

Values are presented as means \pm SD. Results were analyzed using unpaired Student's $t$-tests. Values of $P<0.05$ were considered statistically significant.

\section{Results}

LncRNA KCNK15-AS1 is downregulated in pancreatic cancer tissues and cells

To acquire the transcriptional profile of IncRNAs in pancreatic cancer, paired pancreatic cancer tissues and normal tissues $(n=3)$ were analyzed using ArrayStar IncRNA microarrays. We identified seven candidate lncRNAs (Fig. 1A) that were deregulated significantly between cancer and paired normal tissues. Some of these lncRNAs have previously been investigated in other cancer types, but no published studies have focused on KCNK15-AS1. We examined KCNK15-AS1 expression in 69 paired clinical pancreatic cancer and normal tissues (Fig. 1B). KCNK15-AS1 was downregulated in cancer tissues compared with paired normal tissues. Then, we compared the KCNK15-AS1 expression levels in normal pancreatic ductal epithelial cells (HPDE6-C7) and pancreatic cancer cells (BxPC-3, MIA-PaCa-2, AsPC-1, and PANC1). BxPC-3 and MIA-PaCa-2 had the lowest KCNK15-AS1 expression levels among all cells (Fig. 1C). Thus, we used BxPC-3 and MIA-PaCa-2 to investigate the role of KCNK15-AS1 in pancreatic cancer pathogenesis.

\section{KCNK15-AS1 inhibits cell migration and invasion}

To investigate the regulatory function of KCNK15-AS1, we overexpressed KCNK15-AS1 in BxPC-3 and MIA-PaCa-2 cells (Fig. 2A). Because metastasis is the most malignant feature of pancreatic cancer, we first evaluated cell motility after inducing KCNK15-AS1. The results showed that overexpression of KCNK15-AS1 in MIA-PaCa-2, resulted in significantly reduced migrating and invading cells $(P<0.001)$ (Fig. 2B and $C)$. Similar results were observed for BxPC-3 cells (Fig. 2D and E).

Table 2. Probes used for RNA-FISH

\begin{tabular}{lc}
\hline Probe & Sequence \\
\hline KCNK15-AS1 probe 1 & GCTGCCTTGGGATGCGATGAGTT \\
KCNK15-AS1 probe 2 & TGTATAAGCCTCCCTAACAGAAGCCCA \\
KCNK15-AS1 probe 3 & TCTGGTGGCCACAGTTCTGGAGG \\
\hline
\end{tabular}

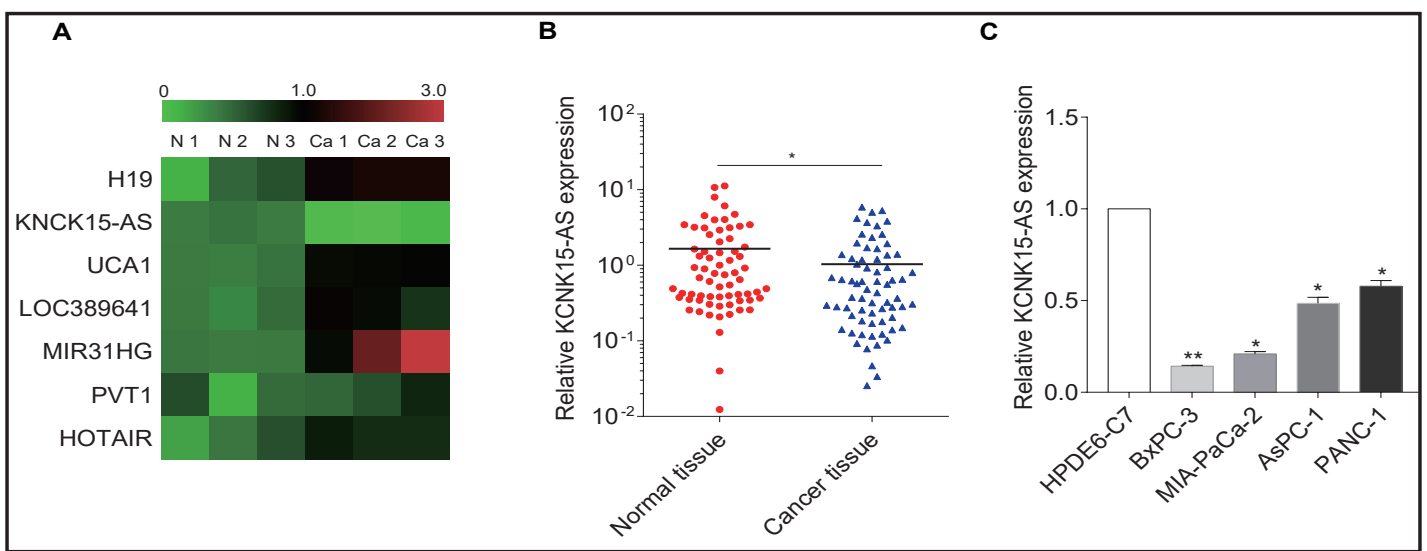

Fig. 1. KCNK15-AS1 is downregulated in pancreatic cancer tissues and cells. (A) Representative result of pancreatic cancer lncRNA microarray analysis. N, Normal tissue; Ca, cancer tissue. (B) In total, 69 paired pancreatic cancer tissues and normal tissues shows significantly decreased KCNK15-AS1 in cancer tissues. (C) Evaluation of KCNK15-AS1 in different pancreatic cells. ${ }^{*} \mathrm{P}<0.05,{ }^{* *} \mathrm{P}<0.01$. IncRNA, long non-coding RNA. 
ALKBH5 demethylates KCNK15-AS1 and inhibits cell migration and invasion Since mRNA m6A modification has been well studied, we considered whether KCNK15-AS1 could be demethylated by ALKBH5. Therefore, we first evaluated KCNK15-AS1 $\mathrm{m}^{6} \mathrm{~A}$ enrichment in HPDE6-C7, MIA-PaCa-2, and BxPC-3 cells. KCNK15-AS1 $\mathrm{m}^{6} \mathrm{~A}$ enrichment was highest in MIA-PaCa-2 and BxPC-3 cells (Fig. 3A). ALKBH5 is a well-characterized RNA $\mathrm{m}^{6} \mathrm{~A}$ demethylase; accordingly, we examined ALKBH5 expression levels in HPDE6-C7, MIAPaCa-2, and BxPC-3 cells. The western blots indicated that ALKBH5 is downregulated in MIA-PaCa-2 and BxPC-3 cells (Fig. 3B). Confocal laser scanning microscopy revealed that the $\mathrm{m}^{6} \mathrm{~A}$ signal colocalized with KCNK15-AS1 (Fig. 3C), indicating that KCNK15-AS1 undergoes $\mathrm{m}^{6} \mathrm{~A}$ modification.

To investigate whether KCNK15-AS1 is regulated by ALKBH5, we overexpressed ALKBH5 in BxPC-3 and MIAPaCa-2 cells (Fig. 4A). As

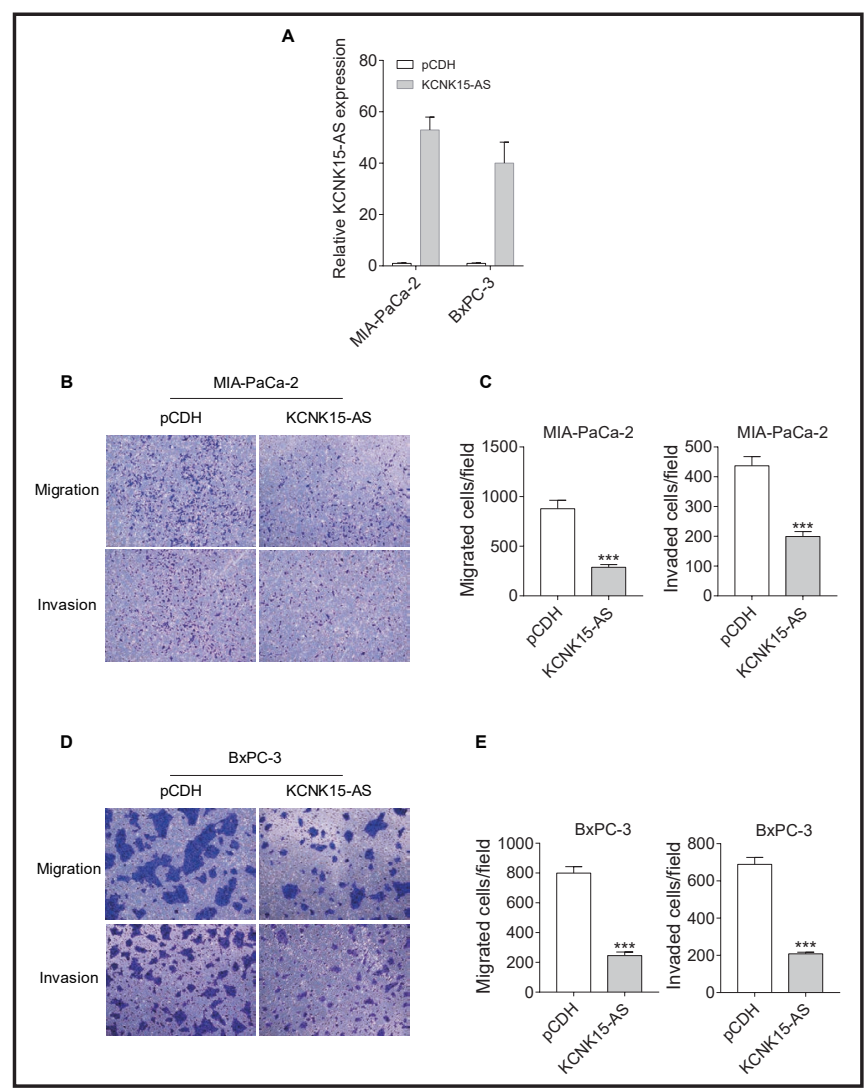

Fig. 2. KCNK15-AS1 inhibits pancreatic cell migration and invasion. (A) RT-qPCR shows successful KCNK15-AS1 overexpression in MIA-PaCa-2 and BxPC-3 cells. (B) KCNK15AS1 inhibits MIA-PaCa-2 migration and invasion (200x magnification). (C) Quantification of the results in (B). (D) KCNK15-AS1 inhibits MIA-PaCa-2 migration and invasion (200x magnification 200×). (C) Quantification of the results in (E). *** $\mathrm{P}<0.001$. RT-qPCR, quantitative reverse-transcription PCR.

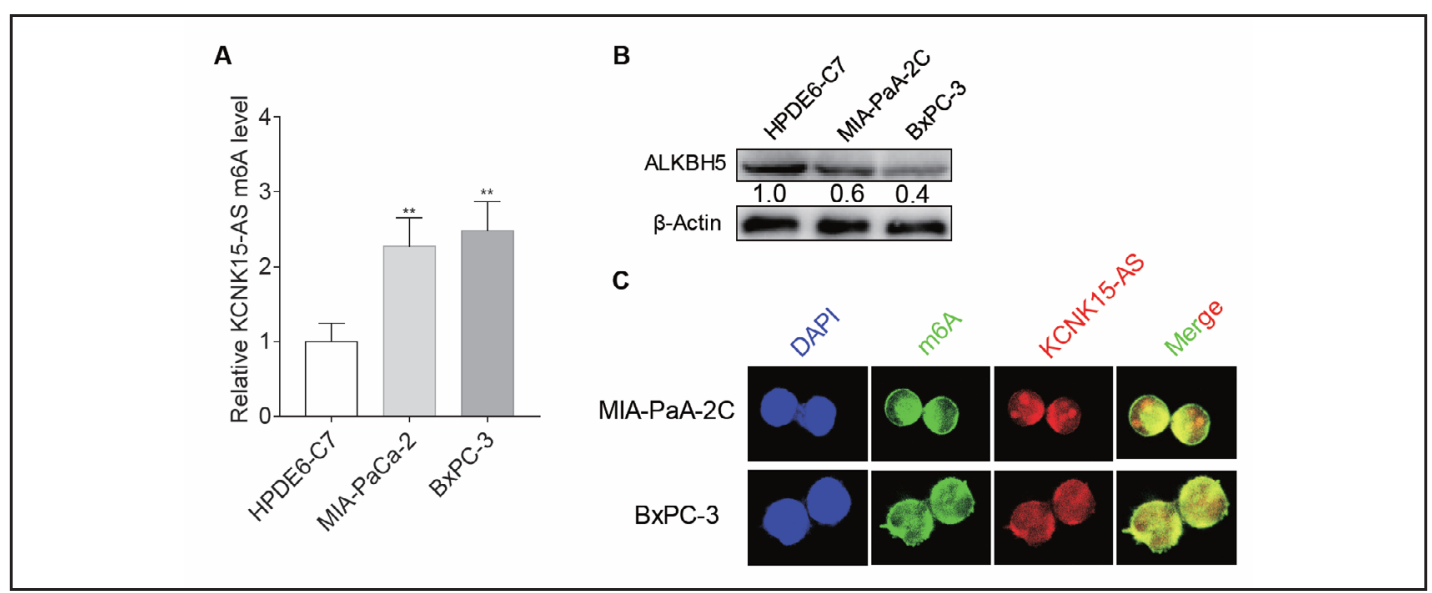

Fig. 3. KCNK15-AS1 undergoes $\mathrm{m}^{6} \mathrm{~A}$ modification. (A) RT-qPCR followed by RNA immunoprecipitation shows that KCNK15-AS1 undergoes $\mathrm{m}^{6} \mathrm{~A}$ modification in various pancreatic cells. (B) Western blotting reveal ALKBH5 expression in various pancreatic cells. (C) Confocal microscopy indicates that ALKBH5 interacts with KCNK15-AS1. RT-qPCR, quantitative reverse-transcription PCR. 


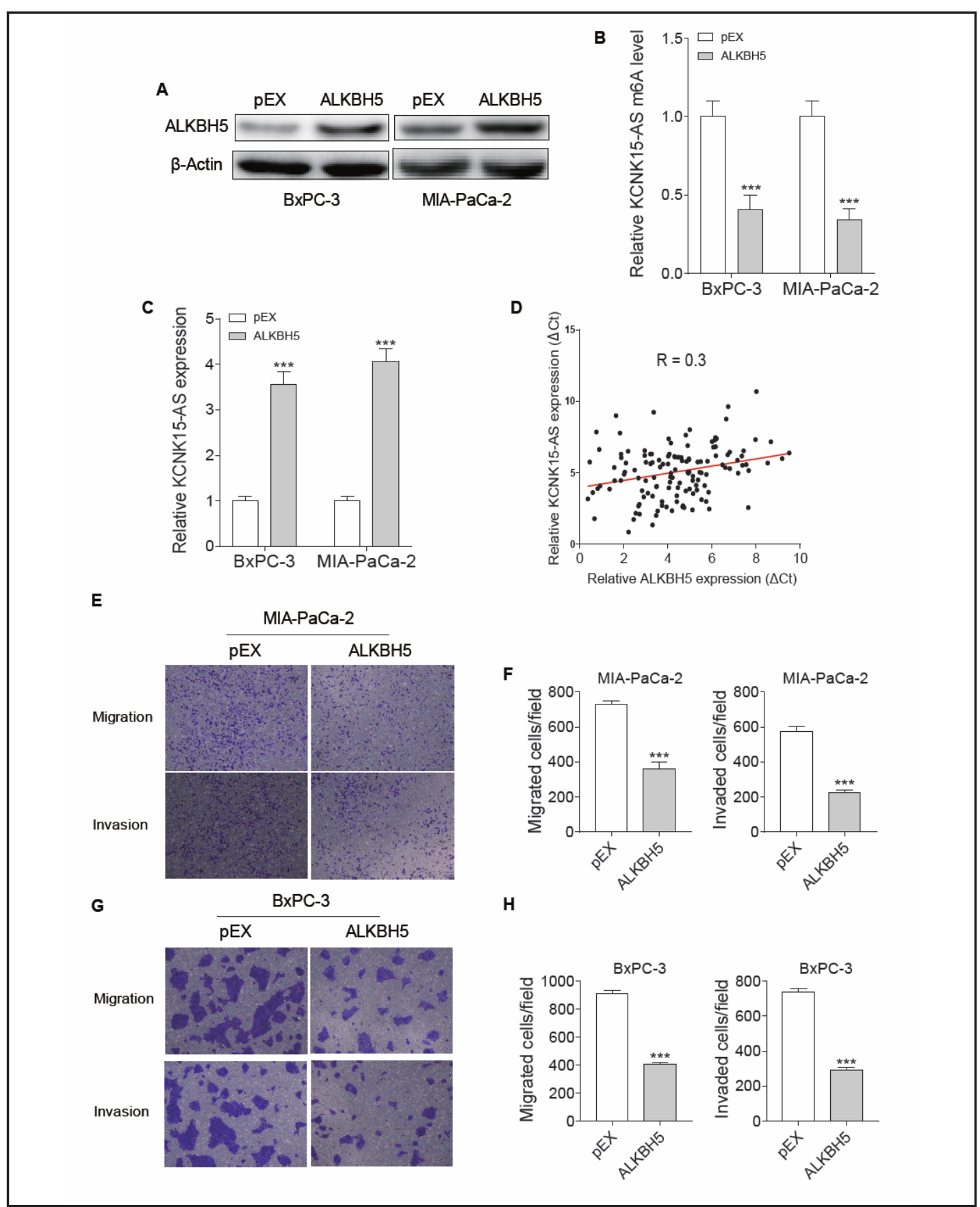

Fig. 4. Influence of ALKBH5 overexpression on KCNK15-AS1-mediated cell migration and invasion. (A) Western blotting reveal that successful ALKBH5 overexpression. (B) ALKBH5 decreases KCNK15-AS1 methylation. (C) ALKBH5 decreases KCNK15-AS1 expression in MIA-PaCa-2 and BxPC-3 cells. (D) ALKBH5 and KCNK15-AS1 are co-expressed in pancreatic tissues. (E) ALKBH5 inhibits cell migration and invasion in MIA-PaCa-2 cells (200× magnification). (F) Quantification of the results in (E). (G) ALKBH5 inhibits cell migration and invasion in BxPC-3 cells (200× magnification). (H) Quantification of the results in (G). *** $\mathrm{P}<0.001$.

expected, the KCNK15-AS1 $\mathrm{m}^{6} \mathrm{~A}$ modification decreased after ALKBH5 overexpression (Fig. 4B). Based on RT-qPCR, KCNK15-AS1 expression was elevated when ALKBH5 was overexpressed in MIA-PaCa-2 and BxPC-3 cells (Fig. 4C). We detected a linear correlation 
between ALKBH5 and KCNK15-AS1 expression in pancreatic cancer tissues (Fig. 4D). Moreover, when ALKBH5 was overexpressed, cell migration (Fig. 4E and F) and cell invasion (Fig. 4G and $\mathrm{H}$ ) both decreased significantly.

Similarly, when silencing ALKBH5 using siRNAs in BxPC-3 and MIA-PaCa-2 cells (Fig. $5 \mathrm{~A}$ ), resulted in increased KCNK15-AS1 $\mathrm{m}^{6} \mathrm{~A}$ enrichment (Fig. 5B), while the expression level of KCNK15-AS1 decreased (Fig. 5C). Additionally, cell migration (Fig. 5D and E) and cell invasion (Fig. 5F and G) increased significantly after ALKBH5 silencing.

\section{KCNK15-AS1 and ALKBH5 inhibit epithelial-mesenchymal transition}

Epithelial-mesenchymal transition (EMT) is associated with cell migration and invasion. We next examined EMT markers in KCNK15-AS1- and ALKBH5-overexpressing cells. The epithelial marker E-cadherin was increased and the mesenchymal markers (E-cadherin and Vimentin) were decreased in KCNK1-AS1-overexpressing cells (Fig. 6A). These results indicate that KCNK15-AS1 inhibits EMT. Similar results were obtained in ALKBH5 overexpression (Fig. 6B) or knockdown (Fig. 6C) cells.

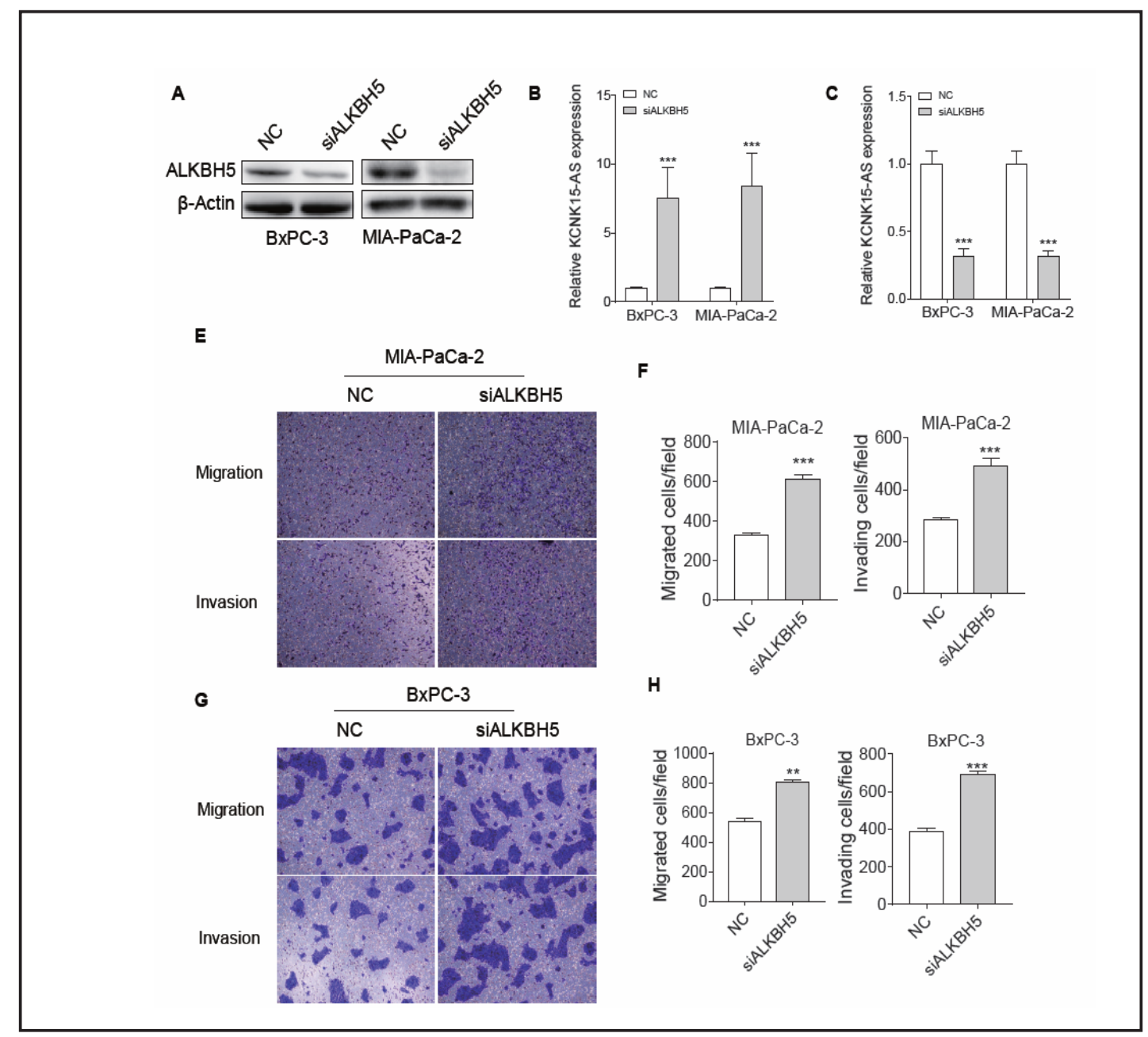

Fig. 5. Influence of ALKBH5 knockdown on KCNK15-AS1-mediated cell migration and invasion. (A) Western blots reveal that ALKBH5 was knocked down successfully. (B) KCNK15-AS1 methylation is increased after ALKBH5 knockdown. (C) Knocking down ALKBH5 decreases KCNK15-AS1 in MIA-PaCa-2 and BxPC-3 cells. (D) Knocking down ALKBH5 promotes cell migration and invasion in MIA-PaCa-2 cells (200× magnification). (E) Quantification of the results in (D). (F) Knocking down ALKBH5 promotes cell migration and invasion in BxPC-3 cells (200× magnification). (G) Quantification of the results in (F). ** $\mathrm{P}<0.01,{ }^{* * *} \mathrm{P}<0.001$.

\section{KARGER}




\section{Cellular Physiology Cell Physiol Biochem 2018;48:838-846 \begin{tabular}{l|l} 
DOI: 10.1159/000491915 & $\begin{array}{l}\text { O } 2018 \text { The Author(s). Published by S. Karger AG, Basel } \\
\text { www.karger.com/cpb }\end{array}$
\end{tabular} ALKBH5 Mediates Pancreatic Cancer via LncRNA}

\section{Discussion}

LncRNAs regulate different aspect of cellular processes including metastasis of cancer cells. Cytoplasmic and nuclear lncRNAs function differently according to the cellular distribution of lncRNAs [9-11]. Cytoplasmic IncRNAs often regulate protein biosynthesis [12], while nuclear lncRNAs tend to affect mRNA transcription and/or alternative splicing $[13,14]$. However, the role of nuclear lncRNAs in epitranscriptional modification remains unclear. In this study, we identified KCNK15-AS1, a IncRNA significantly decreased in pancreatic cancer tissues and inhibited cell migration and invasion.

Recent studies have shown that the $\mathrm{m}^{6} \mathrm{~A}$ mRNA modification is important for the stability mRNA [15], and $\mathrm{m}^{6} \mathrm{~A}$ methylation is a post-transcriptional modification of RNA occurring in the nucleus [16]. RNA $\mathrm{m}^{6} \mathrm{~A}$ methylation is reversible and can be demethylated by ALKBH5 or FTO $[17,18]$. The $\mathrm{m}^{6} \mathrm{~A}$ mRNA modification has been studied extensively; however, the importance of lncRNA methylation has remained elusive [19]. We found that KCNK15-AS1 underwent $\mathrm{m}^{6} \mathrm{~A}$ methylation in pancreatic cells HPDE6-C7, MIA-PaCa-2, and BxPC-3. KCNK15-AS1 could be demethylated by ALKBH5. Moreover, KCNK15-AS1 $\mathrm{m}^{6} \mathrm{~A}$ methylation was negatively correlated with its expression level.

Although ALKBH5 is known to regulate $\mathrm{m}^{6} \mathrm{~A}$ modification [20], the functions of ALKBH5 in pancreatic cells remain unclear. To address this, we overexpressed ALKBH5 in MIA-PaCa-2 and BxPC-3 cells, demonstrating that ALKBH5 could inhibit cell migration and invasion.

In this study, we found a significant decrease in the expression of IncRNA KCNK15-AS1 in pancreatic cancer tissues. This exerted an inhibitory effect on cell motility. Furthermore, KCNK15-AS1 was demethylated by ALKBH5 and was negatively correlated with $\mathrm{m}^{6} \mathrm{~A}$ modification (Fig. 6D). It is possible that $\mathrm{m}^{6} \mathrm{~A}$ could affect the stability of KCNK15-AS1 based on RNA $\mathrm{m}^{6} \mathrm{~A}$ analyses, but the mechanism remains unclear. We also report the novel observation that IncRNA can undergo methylation. We will investigate the potential clinical importance of lncRNA methylation and modifiers of their methylation in future research. Our data could be further developed as a valuable therapeutic strategy for pancreatic cancer prevention and control.

\section{Acknowledgements}

We thank the members of the Dr. Chun Lu laboratory for technical assistance. This work was supported by grants from the National Natural Science Foundation of China (No. 81272382), the Social Development of Science and Technology Research Projects of Jiangsu Province (No. BZ2016788), the Blue Project of Colleges and Universities of Jiangsu Province (No. 2012-16), and the Innovation Capability Development Project of Jiangsu Province (No. BM2015004).

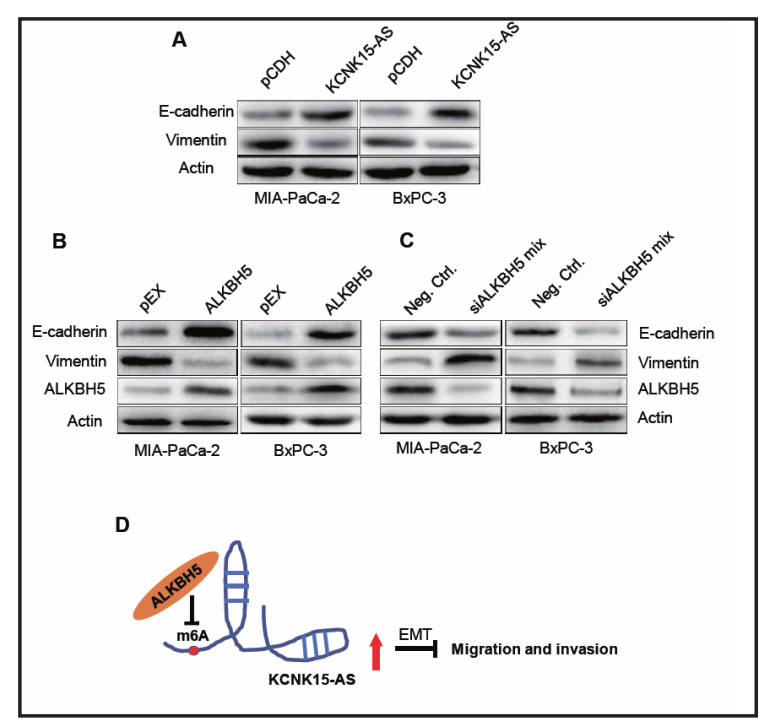

Fig. 6. Inhibitory effects of KCNK15-AS1 and ALKBH5 schematic overview of this work. (A) KCNK15-AS1 nhibits MIA-PaCa-2 and BxPC-3 EMT. (B) ALKBH5 (C) ALKBH5 knockdown promotes MIA-PaCa-2 and BxPC-3 EMT. (D) ALKBH5 inhibits cell motility by demethylating and increasing expression of KCNK15AS1. 


\section{Cellular Physiology Cell Physiol Biochem 2018;48:838-846 \begin{tabular}{l|l} 
DOI: 10.1159/000491915 & $\begin{array}{l}\text { O } 2018 \text { The Author(s). Published by S. Karger AG, Basel } \\
\text { www.karger.com/cpb }\end{array}$
\end{tabular} \\ ALKBH5 Mediates Pancreatic Cancer via LncRNA}

\section{Disclosure Statement}

The authors declare to have no competing interests.

\section{References}

1 Siegel R, Naishadham D, Jemal A: Cancer statistics, 2012. CA Cancer J Clin 2012;62:10-29.

-2 Siegel RL, Miller KD, Jemal A: Cancer statistics, 2015. CA Cancer J Clin 2015;65:5-29.

-3 Sultana A, Tudur Smith C, Cunningham D, Starling N, Neoptolemos JP, Ghaneh P: Meta-analyses of chemotherapy for locally advanced and metastatic pancreatic cancer: results of secondary end points analyses. Br J Cancer 2008;99:6-13.

4 Kim K, Jutooru I, Chadalapaka G, Johnson G, Frank J, Burghardt R, Kim S, Safe S: HOTAIR is a negative prognostic factor and exhibits pro-oncogenic activity in pancreatic cancer. Oncogene 2013;32:1616-1625.

> Li Z, Zhao X, Zhou Y, Liu Y, Zhou Q, Ye H, Wang Y, Zeng J, Song Y, Gao W, Zheng S, Zhuang B, Chen H, Li W, Li H, Li H, Fu Z, Chen R: The long non-coding RNA HOTTIP promotes progression and gemcitabine resistance by regulating HOXA13 in pancreatic cancer. J Transl Med 2015;13:84.

6 Jiao F, Hu H, Yuan C, Wang L, Jiang W, Jin Z, Guo Z, Wang L: Elevated expression level of long noncoding RNA MALAT-1 facilitates cell growth, migration and invasion in pancreatic cancer. Oncol Rep 2014;32:24852492.

7 Machnicka MA, Milanowska K, Osman Oglou O, Purta E, Kurkowska M, Olchowik A, Januszewski W, Kalinowski S, Dunin-Horkawicz S, Rother KM, Helm M, Bujnicki JM, Grosjean H: MODOMICS: a database of RNA modification pathways--2013 update. Nucleic Acids Res 2013;41:D262-267.

-8 Wei CM, Gershowitz A, Moss B: Methylated nucleotides block 5' terminus of HeLa cell messenger RNA. Cell 1975;4:379-386.

-9 Brannan CI, Dees EC, Ingram RS, Tilghman SM: The product of the H19 gene may function as an RNA. Mol Cell Biol 1990;10:28-36.

$>10$ Joung J, Engreitz JM, Konermann S, Abudayyeh 00, Verdine VK, Aguet F, Gootenberg JS, Sanjana NE, Wright JB, Fulco CP, Tseng YY, Yoon CH, Boehm JS, Lander ES, Zhang F: Genome-scale activation screen identifies a IncRNA locus regulating a gene neighbourhood. Nature 2017;548:343-346.

11 Zheng G, Dahl JA, Niu Y, Fedorcsak P, Huang CM, Li CJ, Vagbo CB, Shi Y, Wang WL, Song SH, Lu Z, Bosmans RP, Dai Q Hao YJ, Yang X, Zhao WM, Tong WM, Wang XJ, Bogdan F, Furu K, Fu Y, Jia G, Zhao X, Liu J, Krokan HE, Klungland A, Yang YG, He C: ALKBH5 is a mammalian RNA demethylase that impacts RNA metabolism and mouse fertility. Mol Cell 2013;49:18-29.

12 Gong C, Maquat LE: IncRNAs transactivate STAU1-mediated mRNA decay by duplexing with 3' UTRs via Alu elements. Nature 2011;470:284-288.

- 13 McHugh CA, Chen CK, Chow A, Surka CF, Tran C, McDonel P, Pandya-Jones A, Blanco M, Burghard C, Moradian A, Sweredoski MJ, Shishkin AA, Su J, Lander ES, Hess S, Plath K, Guttman M: The Xist lncRNA interacts directly with SHARP to silence transcription through HDAC3. Nature 2015;521:232-236.

14 Tripathi V, Ellis JD, Shen Z, Song DY, Pan Q, Watt AT, Freier SM, Bennett CF, Sharma A, Bubulya PA, Blencowe BJ, Prasanth SG, Prasanth KV: The nuclear-retained noncoding RNA MALAT1 regulates alternative splicing by modulating SR splicing factor phosphorylation. Mol Cell 2010;39:925-938.

-15 Fu Y, Dominissini D, Rechavi G, He C: Gene expression regulation mediated through reversible m(6)A RNA methylation. Nat Rev Genet 2014;15:293-306.

-16 Lee M, Kim B, Kim VN: Emerging roles of RNA modification: m(6)A and U-tail. Cell 2014;158:980-987.

$>17$ Meyer KD, Jaffrey SR: The dynamic epitranscriptome: N6-methyladenosine and gene expression control. Nat Rev Mol Cell Biol 2014;15:313-326.

18 FTO-Mediated mRNA Demethylation Drives Leukemogenesis. Cancer Discov 2017;7:0F9.

19 Heilmann K, Toth R, Bossmann C, Klimo K, Plass C, Gerhauser C: Genome-wide screen for differentially methylated long noncoding RNAs identifies Esrp2 and lncRNA Esrp2-as regulated by enhancer DNA methylation with prognostic relevance for human breast cancer. Oncogene 2017;36:6446-6461.

20 Zhang C, Samanta D, Lu H, Bullen JW, Zhang H, Chen I, He X, Semenza GL: Hypoxia induces the breast cancer stem cell phenotype by HIF-dependent and ALKBH5-mediated m(6)A-demethylation of NANOG mRNA. Proc Natl Acad Sci U S A 2016;113:E2047-2056. 